\title{
Transient dynamics in non-equilibrium complex systems
}

\author{
Diamantis Sellis \\ sellisd@gmail.com \\ CosmoTech Lyon, France
}

\begin{abstract}
The dynamics of complex systems far from their equilibrium state are currently not fully understood. Besides the theoretical interest for better understanding the world around us this limitation has important practical implications to our ability to model, understand and therefore manage and control complex systems. In a first step to better understand the nonequilibrium dynamics and improve our ability to model complex systems I implement a cellular automaton model of gas mixing. I simulate the evolution towards equilibrium starting from a state of macroscopic order and as the system evolves I calculate the Kolmogorov complexity, the information entropy and the box-counting dimension of the system. I observe a transient peak in complexity, entropy and fractality of the system. To test the genericity of this pattern I implement a very different model, the game of life, where I find the same statistical patterns.
\end{abstract}

\section{Introduction}

Implementing a simulation is an important step in the process of understanding, predicting and controlling complex systems. It often involves modeling multiple interacting entities and processes that span different scales of time and space. For example simulating the evolution of a biochemical pathway requires modeling of entities at very different levels of organization ranging from enzymatic reactions (a process in the order of seconds) to evolving genotypes (a process in the order of hundreds of generations time) [Sellis and Longo, 2014]. Such complex system simulations tend to be computational intensive and hard to scale up. One possible solution is to abstract parts of the model replacing the explicit agent-based modeling by equations or numerical approximations. Unfortunately such abstractions are not always possible as they require an in-depth knowledge of the parts of the system. Even in the cases where such knowledge is available often strong assumptions about steady state have to be made. The search for general patterns in the dynamics of systems out of equilibrium is therefore of great interest and has the potential of revolutionizing the way we model complex systems.

Our current understanding of the non-equilibrium dynamics of complex systems is limited despite the long history of research on the topic[Attard, 2012, Kondepudi and Prigogine, 1998]. However, recently some important steps have been made in understanding the transient dynamics of systems, such as the entropy fluctuations in a system close to equilibrium [Jarzynski, 2011, Evans and Morriss, 2014] and the transient increase in complexity in a mixing system approaching its equilibrium state [Aaronson et al., 2014]. In Aaronson et al. [2014] the authors developed a cellular automaton model, the coffee automaton, to simulate the changes in the apparent complexity during the mixing of liquids. The cellular automaton model aimed to reproduce the "interesting structures" observed during the mixing of milk in a cup of coffee. The authors were the first to report a transient increase in the complexity of the system as it equilibrates. To measure the systems complexity the authors performed a series of transformations including coarse graining and filtering. The multiple specific choices made in each step of the analysis do not permit a conclusive response to how robust the pattern is and most importantly how general it is. Moreover, there is a series of evidence from cognitive science experiments indicating that we tend to consider more "interesting" or "aesthetically pleasing" visual patterns of intermediate complexity [Birkin, 2010]. If indeed the transient increase in complexity is inherent to the dynamics of complex systems transitioning from a state of high level order (far from equilibrium) to a disordered state (equilibrium), then modeling complex systems could greatly benefit.

I here introduce a model of gas mixing starting from a two phase state. The model is inspired by the interacting version of the coffee automaton model [Aaronson et al., 2014]. The design and 
analysis of the gas mixing model overcomes the limitations of the coffee automaton, such as the edge effects and coarse graining. I explore the time evolution of the system as it progressively expands towards equilibrium and measure the time progression of the information entropy, the Kolmogorov complexity, and the fractality of the system at different scales. I then explore the transient dynamics in a completely different cellular automaton model (Game of Life, [Gardner, 1971]). I find across both systems a transient increase in the complexity, information entropy and fractality.

\section{Methods}

I explore the evolution of closed systems towards equilibrium starting from a configuration with a macroscopic structure. To do so I implement two models with very different rules of evolution, a gas mixing model and Conway's game of life [Gardner, 1971]. Simulations start from an initial state far from equilibrium with all particles (or live cells in the case of the game of life) located in a square at the center of the grid. I let the systems evolve towards their equilibrium configuration and measure the systems information entropy (Shannon entropy), Kolmogorov complexity and box counting fractal dimension. I find in both systems a transient decrease in the dimensionality (indicating a fractal structure) and a transient increase in the information entropy and complexity as they approach equilibrium.

\subsection{Topology of the models}

The models are two dimensional cellular automata implemented on a flat torus lattice (periodic boundary conditions) with side $n=2^{6}$. The state of each model can be represented by a square $n \times n$ matrix, where each entry (cell) is an integer. I will refer to the matrix of cells as the "world" of each system. Cells in the topmost row of the matrix are neighboring cells of the bottom row and the same holds true for the first and last column of the matrix. The values of each entry in the matrix is determined based on the rules of each model. The rules are applied iteratively (in discrete time steps here referred to as cycles) leading to the evolution of the system through time.

\subsection{Coarse graining and statistics}

I quantify different aspects of the system using a number of statistical measures. At each cycle in the evolution of the models I coarse grain the world matrix and estimate at different scales the information entropy (Shannon entropy) and the Kolmogorov complexity. I also measure the fractal dimension of the object defined by all cells containing particles of one type in the gas mixing model, or by all live cells in the game of life. The information entropy is a measure of the noise, or randomness, of the system. The Kolmogorov complexity captures the complexity of a system in the sense of its minimal description. The fractal dimension captures how self-similar is the structure of the system across different scales.

The coarse graining involves dividing the system's matrix (the system's world) into nonoverlapping windows of size $w=2^{i}, i \in\{1,2,3,4,5,6\}$. In each window I calculate the sum of all values. Thus, for each window size $w$ a new matrix is generated. I then serialize each matrix into a string $S_{w}$ by concatenating all rows and calculate the information entropy and Kolmogorov complexity.

The information entropy $H$ of a string is defined as:

$$
H=-\sum_{k=1}^{p} \hat{\theta_{k}} \log _{2}\left(\hat{\theta_{k}}\right)
$$

where $\theta_{k}$ is the probability of state $k$ in the string sequence $S_{w}$.

The Kolmogorov complexity $(K)$ of a string is defined as the shortest algorithm that can produce the string [ $\mathrm{Li}$ and Vitányi, 2008]. It is uncomputable, i.e. there is no algorithm that can compute the Kolmogorov complexity of an arbitrary string in finite time [Li and Vitányi, 2008]. However, an upper bound of its value can be calculated by compressing the string. The size of the compressed string and the size of a compressed algorithm that can decompress the string and itself is the upper bound for $K$. I perform PNG compression to get an upper bound for the Kolmogorov complexity of the state of the evolving models. The PNG compression algorithm takes 
into account higher order repeated structures and most importantly the fact that the uncompressed string is a concatenated version of a two dimensional matrix [Duce, 2003]. It is a widely used algorithm for lossless compression of image data. I apply the compression algorithm to the string $S_{w}$ created by concatenating the rows of the coarse grained matrices. The upper bound of the Kolmogorov complexity is the sum of the length of the compressed string and the length of a minimal decompression algorithm (also compressed). Only changes in the Kolmogorov complexity are of interest and not an absolute value, so I only calculate the length of the compressed strings and do not include the length of the decompression algorithms.

The box counting fractal dimension (also known as Minkowski-Bouligand dimension) is a measure of the self-similarity of an object [Mandelbrot, 1982]. It is defined as:

$$
S=\lim _{w \rightarrow 0} \frac{\log N(w)}{\log \left(\frac{1}{w}\right)}
$$

where $N(w)$ are the number of squares required to cover the object of which we measure the dimension, and $w$ is the size of the square. In both the gas free expansion and the game of life models there are two types of cell, cells with value 0 and cells with value 1 . To measure the box counting fractal dimension of the state of the model I define a geometric object composed by all cells with non zero value and measure its dimension. To do so I divide the grid in non-overlapping windows of size $2^{i}$ with $i \in\{1,2,3,4,5,6\}$. For each window size I calculate $N(w)$, the number of windows that overlap at least one cell with non-zero value. If all cells in the grid were filled with 1 s then the ratio $\frac{\log (N(w))}{\log (1 / w)}$ would be 2 , corresponding to the two dimensions of the surface. An object with a fractal structure has a box counting dimension that is not an integer. To accurately determine the box-counting dimension I apply a linear regression model at consecutive window sizes spanning at least 4 window sizes. I select the region with the highest $r^{2}$ value and repeat the process for larger ranges up to the full range of the system. I do not consider a good fit is reached for regions with $r^{2} \leq 0.95$.

\subsection{Initial state and simulation parameters}

All simulations start from a "macroscopic order" configuration, in which a block of side $b=32$ cells is populated with 1 (with a probability of $p=0.9$ ) and all the remaining space with 0 . In this configuration the system has a macroscopic structure and it is far from equilibrium. The simulations proceed in each model depending on its specific rules for a fixed amount of cycles. I empirically determined the equilibration time of each system and set a maximum cutoff Tmax specific to each model.

\subsection{Gas mixing}

The gas mixing is modeled by particles of one type diffusing in a medium filled with particles of a different type (implemented as values of 1 and 0 respectively). The model takes into account interactions between particles by not permitting two particles to be present in the same cell. At each cycle of the simulation a random particle swaps positions with one randomly chosen neighbor particle of opposite type. Each cell has 4 neighbors (diagonal move is not permited). One such move is performed per cycle.

\subsection{Game of Life}

Conway's game of life is an extensively studied two dimensional cellular automaton [Gardner, 1971]. Each cell can have two states and following the historical convention I denote as alive cells those with value 1 and as dead cells those with value 0 . The state of each cell is determined by the states of its 8 neighboring cells and its current state according to the following rules:

1. Live cells with less than 2 live neighbors die.

2. Live cells with 2 or 3 live neighbors remain alive.

3. Live cells with more than 3 live neighbors die.

4. Dead cells with exactly 3 live neighbors becomes alive. 


\subsection{Implementation}

All models were implemented in $\mathrm{C}++$ using the GNU scientific library [Galassi et al., 2009]. The PNG encoding was performed with the lodepng library version 20170917 (http://lodev.org/lodepng/). Statistical analysis and figure generation were performed in R [R Core Team, 2018] using the following packages: tidyverse [Wickham, 2017], latex2exp [Meschiari, 2015], animations [Xie et al., 2017] and rmarkdown [Allaire et al., 2018]. Source code for simulation and analysis is available at https://github.com/sellisd/transientDynamics.

\section{Results}

\subsection{The gas mixing model}

I model mixing of two gases using a two dimensional cellular automaton. The system is initialized with a square block of the grid filled (with a probability of $p=0.9$ ) by particles of one of the two gases and the remaining space is filled by particles of the other type. As the simulation proceeds particles gradually diffuse and mix evenly. The allowed directions of particle movement are 4 (particles cannot move diagonally). To gain an intuitive understanding of the time evolution of the model I depict the system at the initial state, at an intermediate time-point and at equilibrium (Figure 1). As a proxy for the Kolmogorov complexity of the system I perform a PNG compression on the matrix of the system and compute the length of the compressed vector. During a single simulation run I calculate the Kolmogorov complexity of the whole system and of different subregions at regular time intervals. I find that the complexity of the whole system increases through time with small random fluctuations and that there are differences in the complexity of sub-regions (Figure 2).

\subsection{Statistical description of transient dynamics at different scales}

To explore the transient dynamics at different scales I perform a series of simulations starting from a randomized initial state and calculate the median and the $10 \%$ and $90 \%$ quantiles at regular time intervals at different levels of coarse graining. Coarse graining involves dividing the state matrix into non-overlapping windows of size $w=2^{i}, i \in\{1,2,3,4,5,6\}$. In each window I calculate the sum of all values. Thus, to each window size $w$ corresponds a new matrix. The matrix is then serialized into a one dimensional vector $S_{w}$ by concatenating all rows. I calculate the Kolmogorov complexity $(K)$ and information entropy $(H)$ of vector $S_{w}$ and the box counting fractal dimension $(S)$ of the system (Figure 4). I also calculate $\mathrm{K}$ for different subregions of the system (Figure 3). In the initial state the different sub-regions have a very low complexity. The central region, initially filled with particles, has a higher complexity compared to the regions that where initially completely empty.

Small coarse graining window sizes have large $K$ as the length of $S_{w}$ is large. On the contrary the values of $H$ for small coarse graining windows is small as the alphabet size increases for larger $S_{w}$. Focusing on the time evolution of $K$ at the coarse graining window sizes of 4 and 8 we observe a transient increase followed by a gradual decrease of the median complexity (Figure 4). At smaller coarse graining window sizes (2), there is a slight decrease that takes a much longer time, while for larger coarse graining window sizes the system is more homogeneous and fewer changes in complexity are observed. The variance of the complexity is always larger around the peak of the distribution.

Information entropy $H$ is larger for larger coarse graining windows, but for a single coarse graining window during the time evolution of the system its value increases approaching a maximum value given by the size of the window and the average number of cells in the world (Figure 4).

The box counting fractal dimension of the system is transiently sharply reduced. As the system progresses from a filled square towards equilibrium it necessarily passes by an intermediate phase where the particles are distributed forming a gradient of density. The minimum values of the fractal dimension are observed before the peak of entropy and complexity (Figure 4).

\subsection{Genericity}

The observed statistical patterns in the time evolution of the system might be specific to the details of the implemented model. To test the genericity of the observation I implemented a very 
different cellular automaton, the game of life, and repeated the same analysis. In the game of life the total number of live cells (equivalent to particles of type 1 in the gas mixing simulations) is not preserved. The dynamics of the system are very different (Figures S1, S2 and S3) there is a much larger variance in the summary statistics as individual simulation runs are very different from each other (starting from slightly different initial states). Also, there is a pronounced periodicity in the first cycles of evolution. This is due to the rules of the game of life. The initial dense phase is followed by multiple deaths which leads to a relatively lower density and then an increase. Despite the great difference I find here also a transient peak in the complexity and information entropy of the system (Figure 5). The two models share few things in common and these should be responsible for the emergence of the observed statistical patterns during the transition from a high level ordered state to a low entropy state. The most prominent common feature of the two models is that the evolution of the systems necessarily passes by an intermediate state of a density grade. In both cellular automata the rules of evolution are such that particles and living cells respectively cannot move from one part of the grid to a very distant one instantaneously. Therefore, to reach an equilibrium state where particles are more or less evenly distributed in the space starting from a state of macroscopic order (most particles/live cells are in a subregion of the space) it is necessary for the system to pass by a state where the particles (or live cells) form a density gradient that spans a large part of the system. Such gradient has a relatively large complexity, low entropy and high fractality (Figure S4)

\section{Discussion}

I report statistical patterns in the dynamics of complex systems as they transition towards their equilibrium state starting from a state that is far from equilibrium. I find the same patterns in two very different models: the mixing of gases and the game of life [Gardner, 1971]. In both models I find a transient increase in the Kolmogorov complexity, information entropy and fractality despite the great differences in the equilibrium states and rules of each model evolution.

The first report of a transient increase in the complexity of a system as it approaches equilibrium was by Aaronson et al. [2014]. The authors implemented a two dimensional cellular automaton simulation, the coffee automaton. They reported a transient increase in the Kolmogorov complexity of the system, which was measured on the system after a series of transformations such as coarse graining and filtering. The implementation and analysis of the gas mixing model was inspired by the coffee automaton model and it was designed to make more straightforward the measurement of statistical properties such as the Kolmogorov complexity of the system. Despite the implementation differences the two models share some basic similarities and it is possible that these could underlie the observed transient behavior. The other model implemented in the present study, the game of life does not share much with the coffee automaton model. Contrary to the previously analyzed models in the game of life there is no fixed number of particles throughout the evolution of the system. Each cell can have two states (alive:1, dead:0) and there is no restriction on having a total number of alive cells fixed. The fact that the coffee automaton, the gas mixing model and the game of life follow similar transient behaviors strongly indicates that the transient patterns observed across models is a general pattern we might expect to find in other complex systems.

The empirical approach of statistically describing the transient behavior of diverse complex systems can only provide some indications of a general pattern. It is possible that the transient dynamics we observe are limited only to the models tested or some particularity of the parameters explored. It would be of great interest to replicate this approach in other more realistic models and explore their transient dynamics. It is even tempting to consider whether there is a common mechanism causing similar transient behaviors in very different complex systems such as the transient increase in genomic diversity of a population adapting to a new optimum due to clonal interference and the overshooting diversity during an adaptive radiation [Meyer et al., 2011].

If indeed the transient peak in complexity, information entropy and fractality is a general characteristic of complex systems transitioning to equilibrium then this will have a profound impact on our methods for modeling and eventually controlling and managing complex systems. 


\section{Acknowledgements}

I would like to thank Natalia Chousou-Polydouri for comments and feedback of early versions of the manuscript.

\section{References}

Scott Aaronson, Sean M. Carroll, and Lauren Ouellette. Quantifying the Rise and Fall of Complexity in Closed Systems: The Coffee Automaton. page 22, May 2014.

JJ Allaire, Yihui Xie, Jonathan McPherson, Javier Luraschi, Kevin Ushey, Aron Atkins, Hadley Wickham, Joe Cheng, and Winston Chang. rmarkdown: Dynamic Documents for $R, 2018$. URL https://CRAN.R-project.org/package=rmarkdown. R package version 1.10.

Phil Attard. Non-equilibrium Thermodynamics and Statistical Mechanics: Foundations and Applications. Oxford University Press, 2012. ISBN 0199662762.

Guy Birkin. Aesthetic Complexity. PhD thesis, School of Art \& Design at Nottingham Trent University, 102010.

David Duce. Portable network graphics (PNG) specification (second edition). W3C recommendation, W3C, November 2003. http://www.w3.org/TR/2003/REC-PNG-20031110/.

Denis J. Evans and Gary Morriss. Statistical Mechanics of Nonequilibrium Liquids. Cambridge University Press, 2014. ISBN 1107424534.

M Galassi, J Davies, J Theiler, B Gough, G Jungman, P Alken, M Booth, and F Rossi. GNU Scientific Library Reference Manual. Network Theory Ltd., third edit edition, 2009. ISBN 0-9546120-7-8.

Martin Gardner. Mathematical Games. Scientific American, 224(2):112-117, 1971. ISSN 00368733. doi: $10.1038 /$ scientificamerican0271-112.

Christopher Jarzynski. Equalities and Inequalities: Irreversibility and the Second Law of Thermodynamics at the Nanoscale. Annual Review of Condensed Matter Physics, 2(1):329-351, March 2011. ISSN 1947-5454. doi: 10.1146/annurev-conmatphys-062910-140506.

Dilip Kondepudi and Ilya Prigogine. Modern Thermodynamics: From Heat Engines to Dissipative Structures. John Wiley \& Sons, 1998. ISBN 0471973947.

Ming Li and Paul M.B. Vitányi. An Introduction to Kolmogorov Complexity and Its Applications (Texts in Computer Science). Springer, 2008. ISBN 0387339981.

Benoit Mandelbrot. The Fractal Geometry of Nature. W. H. Freeman and Company, 1982. ISBN 0716711869 .

Stefano Meschiari. latex2exp: Use LaTeX Expressions in Plots, 2015. URL https://CRAN. $\mathrm{R}$-project.org/package=latex2exp. R package version 0.4.0.

Justin R Meyer, Sijmen E Schoustra, Josianne Lachapelle, and Rees Kassen. Overshooting dynamics in a model adaptive radiation. Proceedings. Biological sciences, 278(1704):392-8, feb 2011. ISSN 1471-2954. doi: 10.1098/rspb.2010.0640.

R Core Team. R: A Language and Environment for Statistical Computing. R Foundation for Statistical Computing, Vienna, Austria, 2018. URL https://www.R-project.org.

Diamantis Sellis and Mark D Longo. Patterns of variation during adaptation in functionally linked loci. Evolution, 69(1):75-89, jan 2014. ISSN 1558-5646. doi: 10.1111/evo.12548.

Hadley Wickham. tidyverse: Easily Install and Load the 'Tidyverse', 2017. URL https://CRAN. $\mathrm{R}$-project.org/package=tidyverse. $\mathrm{R}$ package version 1.2.1.

Yihui Xie, Christian Mueller, Lijia Yu, and Weicheng Zhu. animation: A Gallery of Animations in Statistics and Utilities to Create Animations, 2017. URL https://yihui.name/animation. $\mathrm{R}$ package version 2.5 . 


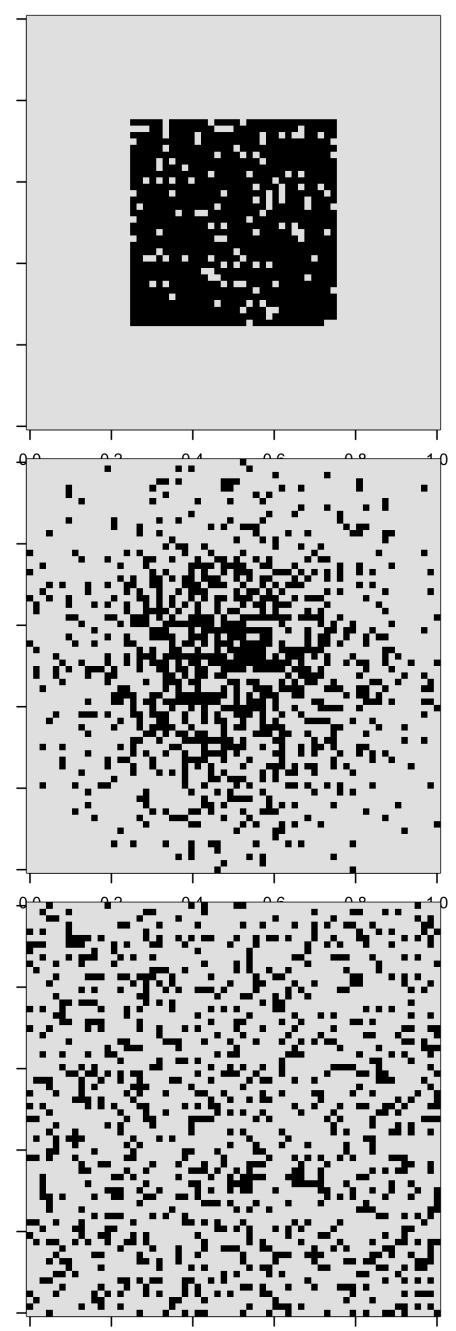

Figure 1: Time evolution of the gas mixing model. The first panel, shows the initial state, the second and third row show the state after $10^{5}$ and $10^{6}$ cycles. Particles diffuse gradually leading to an even distribution of particles across the available space.

Figures 


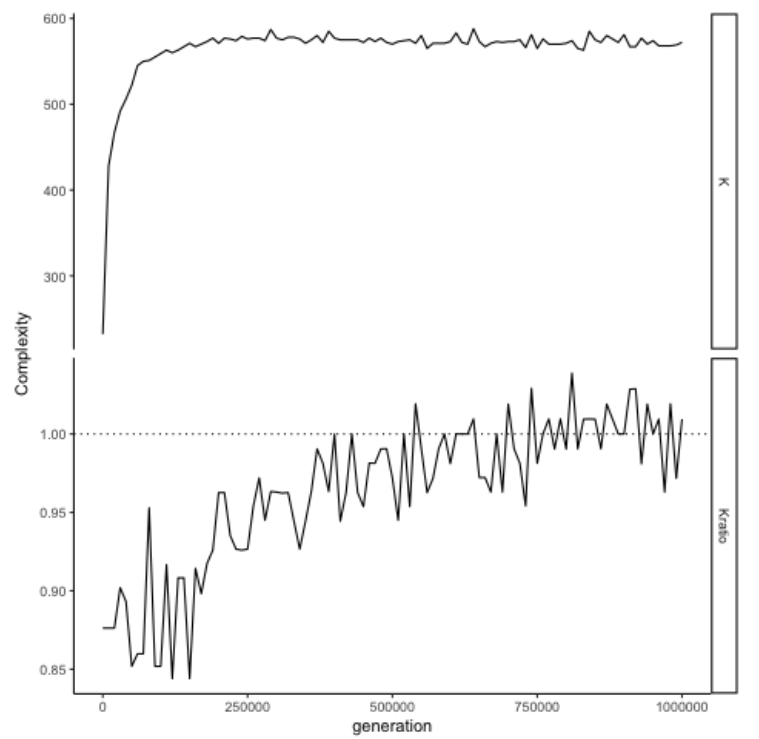

Figure 2: Time evolution of the gas mixing model Kolmogorov complexity during a single simulation run sampled every 1000 steps. TOP: The complexity of the system gradually increases approaching an equilibrium of maximum complexity (disorder). The increase is not strictly monotonic can be seen by the small fluctuations. BOTTOM: Ratio of the Kolmogorov complexity calculated locally for two windows of width 8 cells, at the top left corner and center of the system. Complexity varies at different regions of the space.

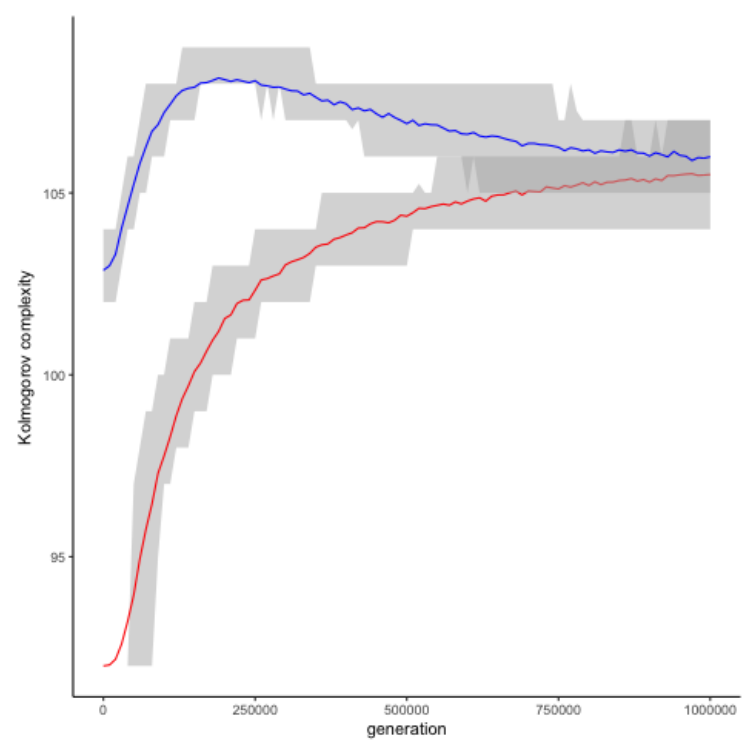

Figure 3: Evolution of the Kolmogorov complexity of two square subregions of the system with side 8 cells averaged across 1000 replicate simulations. Red line corresponds to the top-left corner and blue line to the center. Grey shading represents the first and third quartiles of the distribution. 

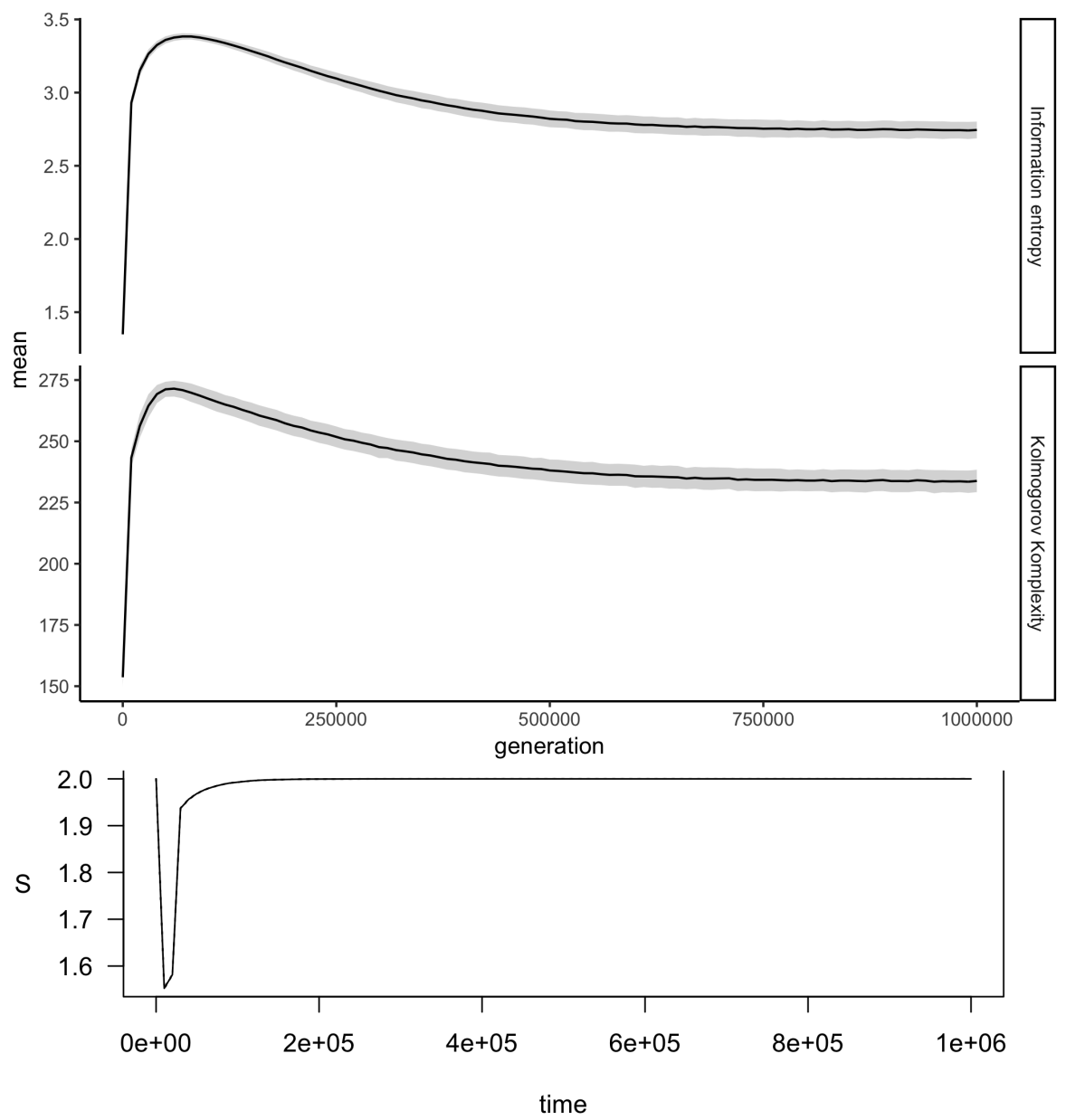

Figure 4: Statistical description of the gas mixing model evolution. Values are computed from an ensemble of 1000 simulation runs. TOP and MIDDLE: Mean (line) and standard deviation (shaded area) of Kolmogorov complexity $(K)$ and information entropy $(H)$ across replicates. A window side of 4 cells has been used for coarse graining BOTTOM: Black line corresponds to the Box-counting dimension $(S)$. 

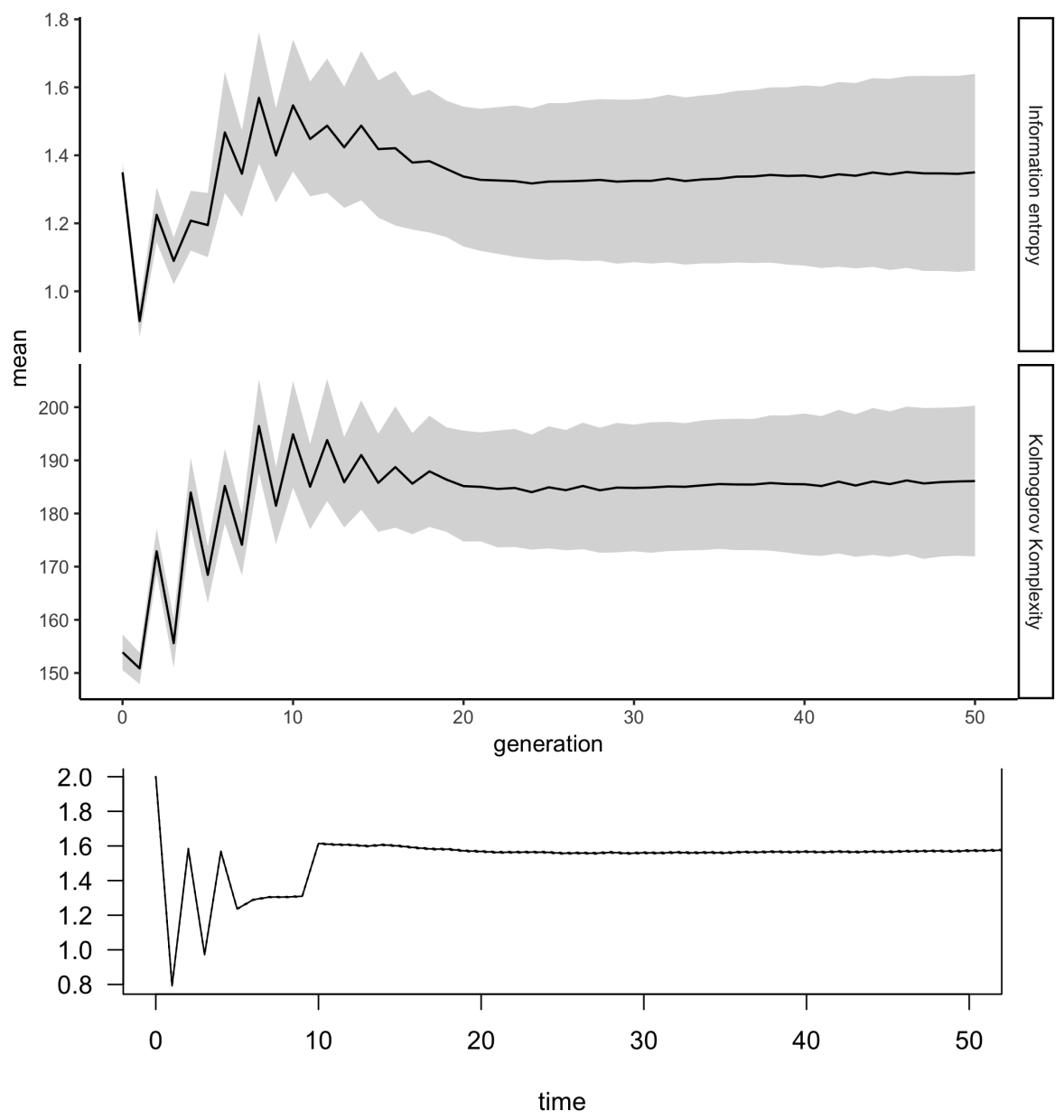

Figure 5: Statistical description of the time evolution of the game of life automaton. Values are computed from an ensemble of 1000 simulation runs. TOP and MIDDLE: Mean (line) and standard deviation (shaded area) of Kolmogorov complexity $(K)$ and information entropy $(H)$ across replicates. A window side of 4 cells has been used for coarse graining BOTTOM: Black line corresponds to the Box-counting dimension $(S)$. 


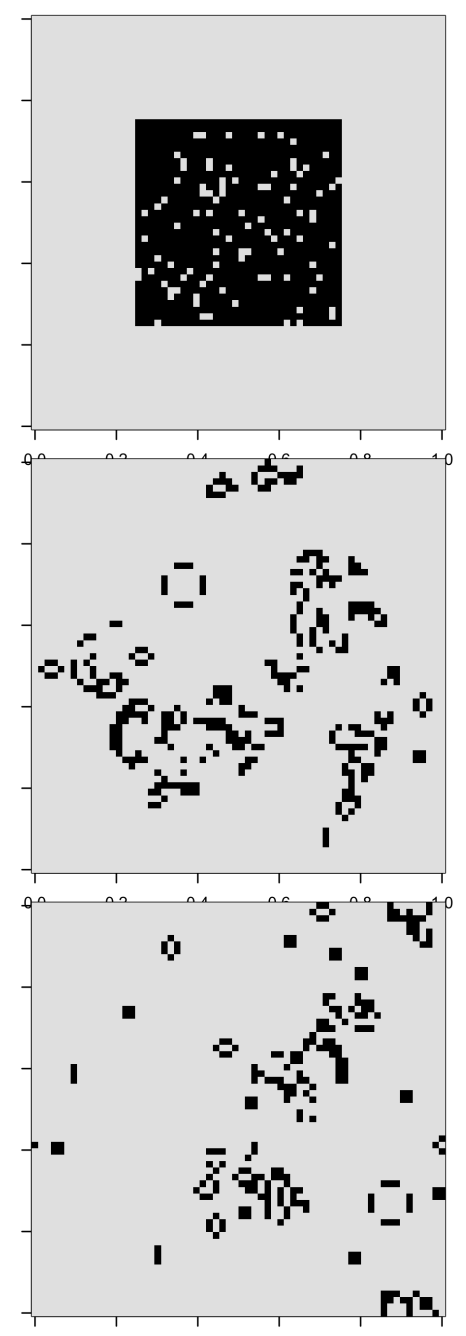

Figure S1: Time evolution of the game of life. The first row, shows the initial state, the second and third row show an intermediate and close to equilibrium state correspondingly (at cycle 10 and 500).

\section{Supplementary Figures}




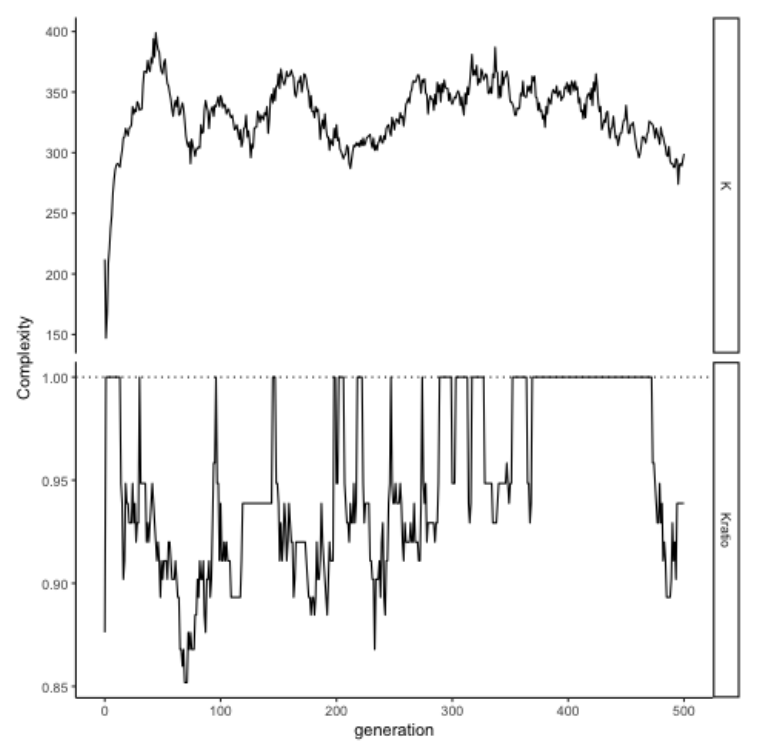

Figure S2: Time evolution of Kolmogorov complexity during a single simulation run of the game of life automaton sampled every 100 steps. TOP: The complexity of the system as it approaches its equilibrium state. BOTTOM: Ratio of the Kolmogorov complexity calculated locally for two windows of width 8 cells, at the top left corner and center of the system. The complexity slightly differs at different regions of the space.

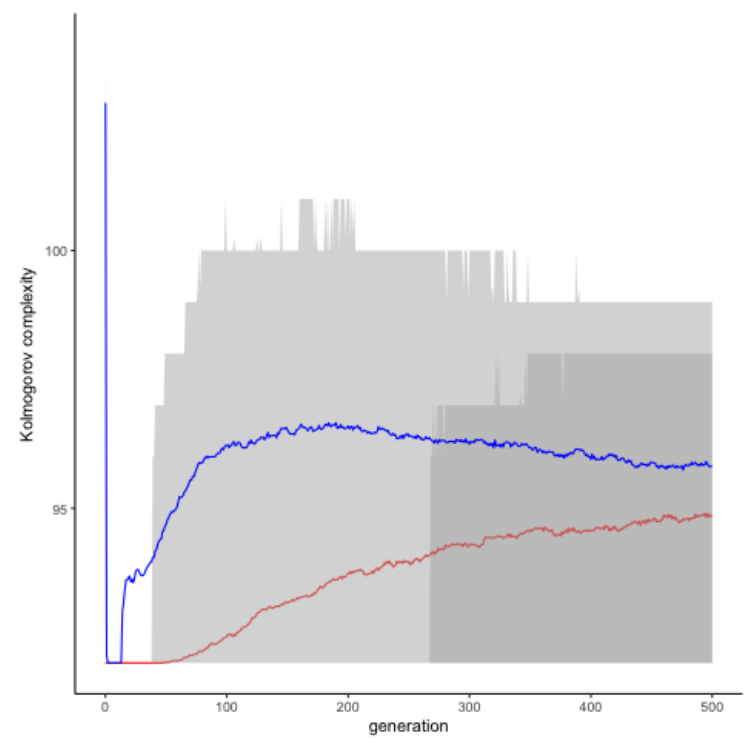

Figure S3: Evolution of the Kolmogorov complexity of two square subregions of the game of life automaton with interactions. Each square has width 8 cells and the values are averaged across 1000 replicate simulations. Red line corresponds to the top left corner and blue line to the center. Grey shading represents the first and third quartiles of the distribution. 


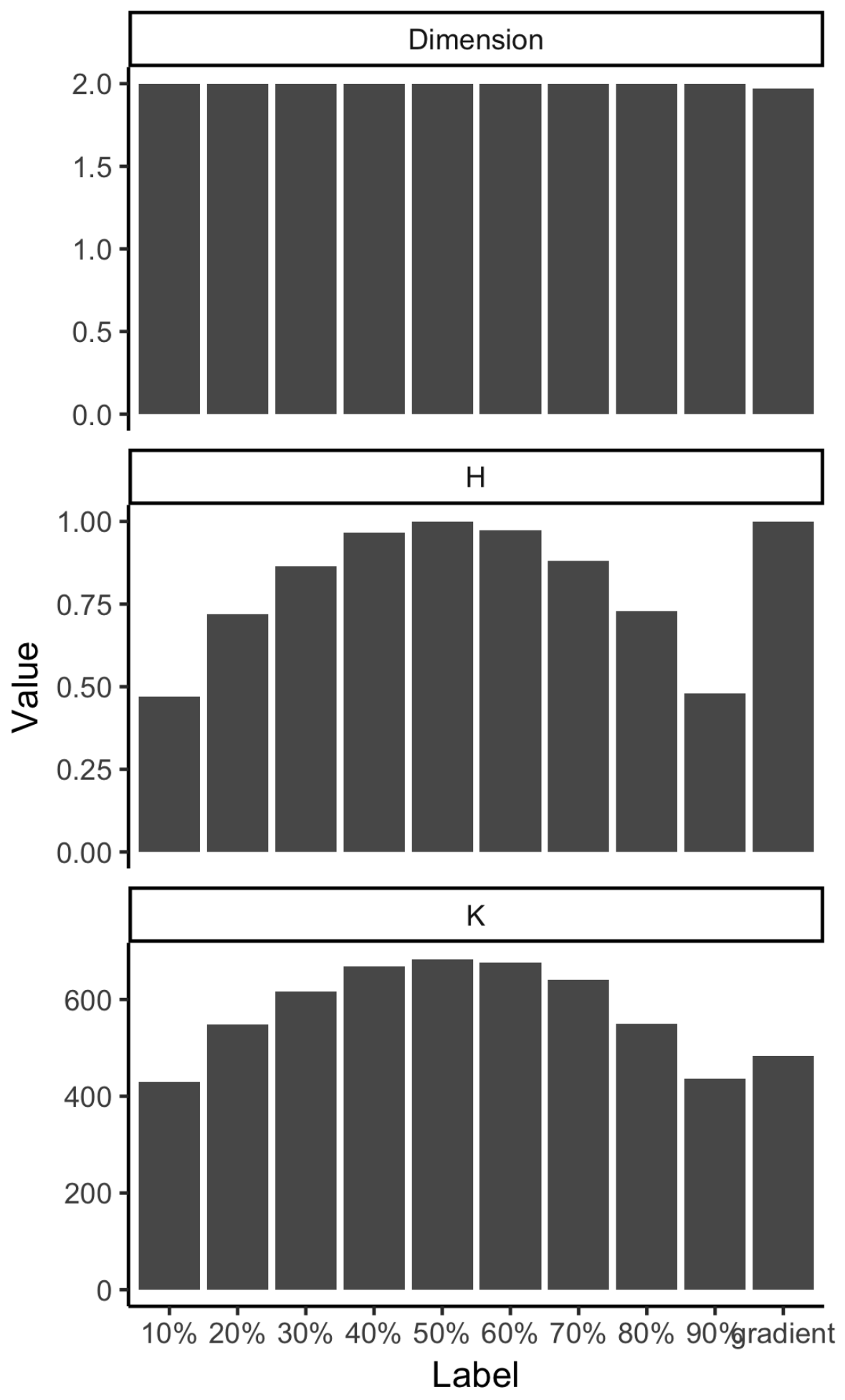

Figure S4: Comparison of the Kolmogorov complexity $(K)$, entropy $(H)$ and fractal dimension of a matrix cellular automaton grid filled with uniform densities or with a density gradient. 